\section{Patch-clampers clean the brain}

\section{Philippe Ascher}

THE emergence of patch-clamping ${ }^{1}$ has undoubtedly been one of the main events in cell physiology in the past 10 years. The most spectacular aspect of this technique was the recording of single-channel currents - the fulfilment of a dream for biophysicists - but it also represents the culmination of a long effort of cell physiologists to achieve control of both extracellular and intracellular solutions. However, there were some limits to the spectacular success of the method when applied to the central nervous system. Because sealing of a patch-clamp pipette requires that the cell surface be both clean (smooth) and visible at high magnification (to allow the delicate 'landing' of the pipette tip), patch-clampers focused their efforts on neurons which could be dissociated and/or kept in tissue culture. With a few exceptions ${ }^{2-4}$ they avoided brain slices which, at the same time, were becoming established as a powerful tool for the in vitro study of central synapses. This barrier is now lifted by the method developed by Edwards et al. ${ }^{5}$, which allows the patch-clamping of neurons in brain slices.

The method devised by Edwards et al. is surprisingly simple and comprises two steps: cutting the slice and cleaning a neuron. The slices are cut thinner (about $150 \mu \mathrm{m}$ ) than the classical slices (about 400 $\mu \mathrm{m})$. This increases the risk of damaging neurons (particularly the delicate, branching dendritic trees), but greatly improves the quality of the image obtained with Nomarski optics. The use of thin slices has previously been advocated as a way to improve the identification of neurons $\mathrm{s}^{6}$ and is one of the key advantages of organotypic slices $^{7}$. Note, however, that any new method of improving visualization of the surface could relax the requirement for

100 years ago

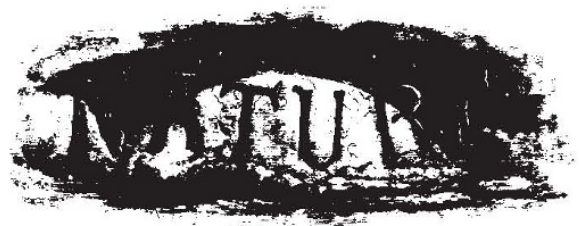

An Earthquake?

ON Friday, July 5, the inhabitants of Lyme Regis were much astonished by some noises, which took place at intervals between 11 and 11.15 p.m., and which there seems good reason to believe were caused by an earthquake. In three houses the occupiers thought that heavy pieces of furniture were being moved about, which was of course found not to be the case; and in another the inmates thought at first that something was wrong with the kitchen boiler. thinness and allow the use of classical slices.

The second step is the cleaning, which is done without enzymes (see figure). Takahashi noted a few years ago that blowing a stream of physiological solution onto a neuron from a broken pipette was often sufficient to remove the debris covering the surface of the neuron. Edwards $e t$ $a l .^{5}$ find that cleaning is improved if alternated with suction, which prevents debris from being deposited. Because the cleaned surface can be restricted to a few square micrometres, the neuron under study will not float away from the slice as it can do after enzymatic treatment.

The new method can in principle be applied to slices taken from any region of the brain, and thus should allow patchclamping of any neuron provided that it can be properly identified and is not covered by a non-neuronal cell. In addition, the method allows the study of identified synapses which can be activated by stimulation of identified tracts but also, it seems, by direct stimulation of a presynaptic neuron. This should allow analysis of quantal mechanisms, of potentiation, of depression and so on, in unrivalled conditions of control of the extra- and intracellular solutions. The new method should, furthermore, end the debate between 'patch-clampers', who advertised the fine resolution of their techniques, and 'slicers', who stressed that cultured neurons are often poorly identified, possibly de-differentiated, and that the synapses that form in culture may have little in common with those in a normal brain.

Will patch-clamping in slices provide an answer to outstanding questions in in vitro electrophysiology of the central nervous system? Some serious problems remain.

The noises observed consisted of a distant rumble which grew nearer till at last the windows of the houses rattled, and in some cases distinct vibrations of the houses were felt. Some have supposed that these noises were caused by guns at sea, but this seems impossible, because (1) the rattling of the windows occurred after the distant rumble, and not simultaneously as would have been the case with guns; (2) a gentleman who has had much experience in guns and firing, has declared that the noise was not like guns; (3) after making enquiries we have been unable to discover that any firing at sea took place that night; (4) although the night was still, a heavy ground swell was observed. These phenomena have not received any notice as far as we know in the public press, and it seems a pity, if an earthquake, as we believe, really took place, that there should not be some record of it.

A. R. SHarpe.

From Nature 40, 294; 25 July 1989.

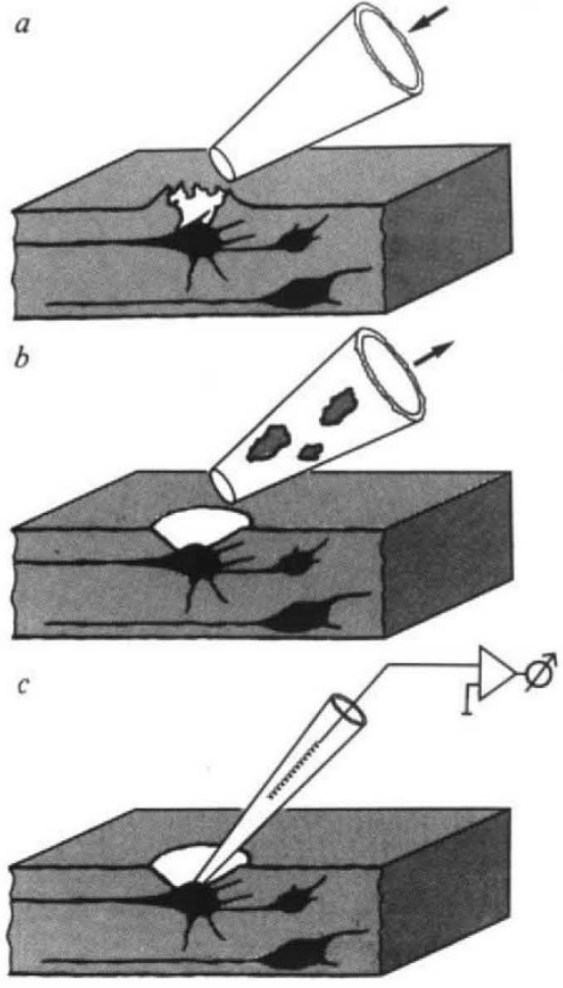

Patch-clamping involves the use of a glass micropipette $(0.5 \mu \mathrm{m}$ diameter) filled with current-conducting saline solution, into which a small patch of plasma membrane, which must be clean, is sucked. The patch membrane is depolarized and clamped at a set voltage and the flow of ionic current across the patch monitored by an electrode connected to an amplifier. The new technique of Edwards et al. ${ }^{5}$ involves cleaning the surface of a neuron contained in a slice by alternately blowing (a) and sucking (b) solution through a pipette placed above the target. The patch pipette can then be sealed onto the exposed neuronal surface (c). (Courtesy of Dr B. Sakmann.)

First, as patch-clamping implies replacement of intracellular media by artificial solutions, it is possible that critical intracellular components are washed away. In some cases, these factors can be added back by placing cellular extracts on the internal surface ${ }^{8}$. In other cases, washout can be minimized by methods such as the perforated whole-cell method ${ }^{9,10}$. Washout may also be a problem in the extracellular space, because both the thinning of the slice and the cleaning of the neuronal surface may open the extracellular space in an unphysiological way, depleting important extracellular molecules or preventing their accumulation. The problem may be less severe, however, inasmuch as the method of cleaning can restrict the exposed surface.

Finally, the most difficult problem could be to control the voltage of neuronal processes such as dendrites and axons. Dendrites are probably the main site of transmission at excitatory synapses, but synapses are also known to occur on axons. Predicting what happens in distant 